\title{
Differences in the Reasons of Intermittent versus Permanent Discontinuance in Social Media: An Exploratory Study in Weibo
}

\author{
Zhongyun Zhou \\ School of Economics and \\ Management, Tongji University \\ philzhou@tongji.edu.cn
}

\author{
Mingzhu Yang \\ School of Economics and \\ Management, Tongji University \\ yangmingzhu@tongji.edu.cn
}

\author{
Xiao-Ling Jin \\ Information Manegement \\ Department, \\ Shanghai University \\ $\underline{\text { Kathyjin2011@shu.edu.cn }}$
}

\begin{abstract}
Discontinued usage (or discontinuance) of social media has aroused extensive interests among researchers and practitioners. Existing research tends to view discontinuance as permanent, but pays little attention to intermittent discontinuance wherein individuals stop using a social medium for a certain period of time but eventually restart using afterwards. Specifically, little is known about the commonalities and differences in reasons underlying permanent and intermittent discontinuance. Addressing this research gap, this paper reports an exploratory field study using qualitative data collected by interviewing 96 users with discontinuance experiences in Weibo, a popular Chinese microblogging platform. Results reveal four categories of reasons for discontinuance: user-, context-, function- and content-related. Comparative analysis shows that user discontinuance because of context-related factors is more likely to be intermittent, while user discontinuance due to function-and content-related factors are more likely to be permanent; results for user-related factors are complicated. Theoretical and practical implications are discussed.
\end{abstract}

\section{Introduction}

Social media are computer-mediated technologies that enable users to present themselves via an online profile shared with their friends and encourage them to get involved in various online activities including sharing, co-creating, discussing, and modifying usergenerated content $[1 ; 2]$. The widespread use of social media has profoundly transformed e-commerce into social commerce $[3 ; 4 ; 5 ; 6]$. According to a recent report by China Internet Network Information Center (CNNIC) [7], social media have become ubiquitous and important in Chinese people's daily life to learn about their friends, get and share diverse information, shop online, and even complete payment. The number and activeness of users are critical for the success of social media and the achievement of their business values [8; 9].

However, social media are in a dilemma where many users reduce their activeness and even stop their usage in recent years. According to the abovementioned CNNIC Report [7], the number of Chinese netizens who use Weibo and social media alike has been continuously decreasing from 309 million in January 2013 to 230 million in January 2016. As a consequence, a number of social media platforms have stopped their operations or shut down part of their functions; this forced many companies conducting social commerce on these platforms to get back to expensive traditional media, thereby reducing corporate profits [10]. Therefore, users' discontinuance has become a core issue for the sustainable development of social media and social commerce. Thus, it is important to understand the mechanisms driving user discontinuance, an issue that has been rarely examined.

The existing literature on discontinuance tends to view discontinuance of an innovation as a permanent end of its life cycle, assuming (implicitly) that discontinuers will never come back. This view, although valuable, is incomplete by ignoring the fact that many discontinuers may later on readopt the innovation, a phenomenon called intermittent discontinuance $[11 ; 12 ; 13 ; 14]$. Intermittent discontinuance means that in the post-adoption stage of an innovation, a subject decides to or is forced to stop using the innovation for a certain period of time but will eventually re-adopt it afterwards. In other words, intermittent discontinuers may be trapped in the circle of "stop" and "re-adoption" but never give up using the innovation entirely.

Despite the recently increasing research interests on discontinuance and the conceptualizations of intermittent vs. permanent discontinuance, little (if 
any) research has explored the commonalities and differences in the driving mechanisms underlying these two types of discontinuance. Specifically, intermittent and permanent discontinuance both start with users' quitting but evolve different afterwards, in the sense that intermittent discontinuers will come back sooner or later. If social media operators can understand the different reasons underlying intermittent versus permanent discontinuance, they can make better predictions at the very beginning of discontinuance about whether the discontinuance will be permanent according to the stated reasons. Moreover, they can make interventions appropriate to the specific factors that are found to be more important in driving discontinuance (especially permanent discontinuance). Thus, it is important to consider both types of discontinuance simultaneously in a single study, in order to build a systematic understanding of the commonalities and differences in the driving mechanisms underlying these two types of discontinuance. However, the existing research on discontinuance tends to focus only on one type of discontinuance (mostly permanent discontinuance). To fill this gap, this study aims to address two research questions (RQ):

RQ1: Why do individuals discontinue using a social medium, either permanently or intermittently?

RQ2: What are the commonalities and differences in reasons underlying permanent and intermittent discontinuance?

In order to address these research questions, this paper reports an exploratory field study of users in a popular Chinese microblogging platform. Data are collected from in-depth interviews of 96 users with discontinuance experiences and diverse backgrounds. Content analyses of the qualitative data and subsequent statistical analyses suggest that intermittent and permanent discontinuance share some common antecedents but also occupy unique reasons. As such, we evidently show that permanent discontinuers can indeed be distinguished from intermittent discontinuers in terms of reasons of discontinuance. Accordingly, the results of this study will help social media providers to deal better with the two types of discontinuance, especially by attending to addressing those factors driving permanent discontinuance.

The rest of the paper proceeds as follows. An overview of the previous research on discontinuance in information systems is presented at first. This is followed by a description of the research method and report of results. The final section discusses the implications of this study for research and practice, and suggests avenues for further research.

\section{Literature review}

\subsection{Permanent discontinuance and intermittent discontinuance}

Discontinuance was initially introduced to empirical research by Rogers, defined as "a decision to reject an innovation after it has previously been adopted during the confirmation stage" [15]. According to Rogers' Diffusion of Innovations (DOI) theory, adopters who already made the decision seek reinforcement at the confirmation stage and may reverse the decision if exposed to conflicting messages regarding the innovation [15]. Discontinuance occurs when an organization ceases the use of an information system [16] or an individual decides "to stop using an information system in support of a work task" [17]. Specifically, an individual may decide to replace an inferior innovation with a superior one (replacement discontinuance) or discontinue with an innovation when there is dissatisfaction with its performance (disenchantment discontinuance) [18]. Overall, these early definitions of discontinuance tend to view discontinuance as a one-time decision of stopping using an information system at the end of its life cycle.

However, the latest progress in this area suggests that discontinuance can be more complex than defined above. First, discontinuance does not occur suddenly but may evolve through a process characterized by short breaks, control activities and suspend behavior [19]. As a consequence, discontinuance is not a flip-side of continuance but can exert through a spectrum of multiple behaviors including decreasing use intensity, stopping use temporarily or permanently, or switching to alternatives [20]. Individuals who intentionally reduce their usage intensity are still continuous users literally but may have the potential to become discontinuers in the future. Thus, discontinuance is not necessarily a one-time decision of complete abandon of the information system in use.

Second, discontinuance does not necessarily represent the end of life cycle of the innovation but can be just one phase in the post-adoption stage. By taking the time dimension into consideration, scholars have differentiated two types of discontinuance behaviors, namely, permanent discontinuance and intermittent discontinuance [14]. While the former usually means a permanent cessation of use, the latter represents only temporary breaks from the innovation [14; 19]. Discontinuance behavior is considered permanent if the discontinuers do not come back any more [12; 14]. Yet, if an individual who has stopped using the innovation 
Table 1. Literature review of discontinuance in information system

\begin{tabular}{|c|c|c|c|c|c|}
\hline \multirow{2}{*}{$\begin{array}{l}\text { Reference } \\
\text { Number }\end{array}$} & \multirow{2}{*}{$\begin{array}{c}\text { Type of } \\
\text { Information System }\end{array}$} & \multicolumn{4}{|c|}{ Antecedents } \\
\hline & & User & Context & Function & Task \\
\hline$[30]$ & Online services & & $\begin{array}{l}\text { Interpersonal Influence ; External } \\
\text { Influence }\end{array}$ & & \\
\hline [31] & Software Application & Experience Types & Social Effects & Technical Effects & \\
\hline$[24]$ & Organizational IS & Discontinuance Inertia & $\begin{array}{l}\text { Stakeholder Support; Mimetic } \\
\text { Isomorphism }\end{array}$ & & \\
\hline [21] & Social Network Sites & $\begin{array}{l}\text { Perceived needs; Personal } \\
\text { innovativeness }\end{array}$ & Perceived Popularity & Characteristic & \\
\hline$[23]$ & Organizational IS & $\begin{array}{l}\text { Organizational Growth; } \\
\text { Organizational Change; } \\
\text { System Investment; System } \\
\text { Embeddedness }\end{array}$ & $\begin{array}{l}\text { Competitive Change; Regulatory Change; } \\
\text { Changing Customer Needs; Supply Chain } \\
\text { Changes; } \\
\text { Technological Change; Mimetic } \\
\text { Isomorphism }\end{array}$ & & \\
\hline$[16]$ & Organizational IS & $\begin{array}{l}\text { System Invest; System } \\
\text { Embeddedness }\end{array}$ & $\begin{array}{l}\text { Environmental Change; Institutional } \\
\text { Pressures }\end{array}$ & $\begin{array}{l}\text { System } \\
\text { Shortcomings }\end{array}$ & \\
\hline$[17]$ & Individual IS & Perceived Sunk Costs & Perceived Work; Impediment & $\begin{array}{l}\text { Ease of Use; } \\
\text { Usefulness }\end{array}$ & \\
\hline$[22]$ & Social Network Sites & $\begin{array}{l}\text { Self-Efficacy to Discontinue } \\
\text { Guilt Feelings; } \\
\text { Habit of Using the Site; }\end{array}$ & & & \\
\hline [29] & Social Network Sites & & Social Overload & & \\
\hline$[27]$ & Social Network Sites & $\begin{array}{l}\text { Pattern; Transition Costs; Sunk } \\
\text { costs; replacement }\end{array}$ & Invasion, Disclosure, Social Overload & $\begin{array}{l}\text { Complexity, } \\
\text { Uncertainty }\end{array}$ & \\
\hline [28] & Social Network Sites & & Technical Disturbance; Social Disturbance & & \\
\hline$[26]$ & Social Network Sites & & Social Overload & $\begin{array}{l}\text { Feature } \\
\text { Overload }\end{array}$ & $\begin{array}{l}\text { Information } \\
\text { overload }\end{array}$ \\
\hline [14] & Social Network Sites & $\begin{array}{l}\text { Burden on Time/Resources; } \\
\text { Lack of Use; Privacy concerns; } \\
\text { Confused; Religion }\end{array}$ & $\begin{array}{l}\text { Replacement with Other Innovation; } \\
\text { Physically Unavailable; }\end{array}$ & & $\begin{array}{l}\text { Disenchantment } \\
\text { with Content }\end{array}$ \\
\hline$[13]$ & Smart Health Device & $\begin{array}{l}\text { Attitude Instability; } \\
\text { Emotional Valence Fluctuation }\end{array}$ & & & \\
\hline
\end{tabular}


readopt it later on, then his/her discontinuance behavior should be considered intermittent [11]. In other words, this study focuses on those who have discontinuance experience. If one has never readopted the innovation since his/her first discontinuance, he/she is regarded as a permanent discontinuer; whereas those who had experience to come back are viewed as intermittent discontinuers.

Research on intermittent discontinuance is just emerging. Zhang and Ye differentiated the adopters that exhibit the pattern of adoption-discontinuancereadoption from other categories of adopters [12]. In the context of social media, York et al. coined the phenomenon of "Facebook vacation" and explored the reasons underlying temporary discontinuance on the basis of secondary data [14]. Further, Shen and Li developed a model to explore the effects of attitudinal ambivalence and emotional arousal on intermittent discontinuance in the context of smart health devices [13].

Despite these recent research endeavors on intermittent discontinuance, a systematic investigation on this phenomenon with comparison to permanent discontinuance is still lacking. Especially, given the conceptual differences between intermittent and permanent discontinuance, little is known about the commonalities and differences between them in terms of their underlying reasons and mechanisms.

\subsection{Reasons for discontinuance}

We review the existing literature on discontinuance and summarize the antecedents of discontinuance suggested in the literature. As shown in Table 1, these factors can be categorized into four types.

First, user-related factors are key antecedents of discontinuance of innovation. Individual-level userrelated factors include user's habit, time limitation, satisfaction, guilty feelings, and so on $[13 ; 14 ; 20 ; 21$; 22], while organizational-level factors include group growth, system investment, and embeddedness [16; $23 ; 24 ; 25]$. Second, some studies shed light on context-related factors such as social effects, technical disturbance, and replacement with other innovation $[14 ; 26 ; 27 ; 28 ; 29]$. The third general category refers to functional factors such as system shortcomings, complexity, and uncertainty $[16 ; 20$; $21 ; 23 ; 26]$. Finally, a few studies have mentioned task or, in the context of social media, content-related factors $[14 ; 20 ; 26]$.

In sum, although prior research has suggested a number of potential antecedents of discontinuance, there are still several important research gaps. First, few studies have offered a comprehensive or systematic investigation of the antecedents of discontinuance. Most of them focus only on a small set of factors, making it difficult to understand the importance of different factors that drive discontinuance behavior. While some factors are well examined, others (e.g., task-related factors) are less considered in the present literature. Second and more importantly, the existing literature tends to investigate only one type of discontinuance (mostly permanent discontinuance). To our knowledge, little (if any) has considered both types of discontinuance at the same time. As such, we are unable to know the commonalities and differences in the antecedents of intermittent versus permanent discontinuance. The next sections report our exploratory field study aiming to address these issues.

\section{Method}

\subsection{Data collection and participants}

Semi-structured interviews were conducted in order to explore individual reasons for discontinuance behavior in online social media. Sina Weibo was chosen as the research site as it is leading the microblog market in China and can represent the typical development of information-driven online social networks [32]. Just like Twitter, Sina Weibo provides microblogging services which allow people to publish, share and discuss short messages on the Web. Twitter inspired Sina Weibo, but the latter has now gained a greater popularity and larger population of users. By the end of March 2017, the number of monthly active users in Weibo reached 340 million, which has exceeded that of Twitter.

A snowball sampling method was used to identify potential subjects for this study. While random sampling is not a necessity for interview studies like this one, we purposefully recruited subjects with diverse demographics and usage experiences as much as possible. Each participant in our interview was guaranteed for anonymity and privacy protection, and asked to suggest a group of three to five other potential participants at the end of the interview. Eventually, we were able to recruit 200 subjects willing to participate in the semi-structured interviews which permit in-depth exploration of the research questions with each study participant.

We interviewed the subjects face to face wherever possible; for those subjects located remotely, we used instant messaging tools with audio chatting functions or telephones to conduct the interview. The language for interviews was Chinese. All interviews were taperecorded upon the subjects' consent, and transformed 
into textual forms afterwards by a group of ten welltrained research assistants with cross-validation.

The interview was conducted following an interview protocol which involved three parts. In the first part, we asked the subject to report his/her usage experience with Weibo in order to pick out those of interest (i.e., "Have you used Sina Weibo?", "When did you start using it?", "Are you still using it now?", and "Have you ever stopped using it?"). Among the 200 contacted subjects, only 96 had experience of discontinuance; they were qualified for this study and asked to answer our remaining questions.

Second, each qualified subject was then asked to describe the comprehensive reasons of discontinuance ("Why did you stop using Sina Weibo?") and, in the case of intermittent discontinuance, the reasons of re-adoption ("Why did you readopt Weibo afterwards?"). The subject was encouraged to report as many reasons as possible while the issues were suggested by our priori framework. Finally, he/she was also asked to report his/her demographic factors including age, gender, education level, job, and industry.

Among the 96 qualified subjects in our sample, $53(55.21 \%)$ were male and $43(44.79 \%)$ were female. The age structure of our sample is as follows: below 20 years of age $(4.17 \%), 11-25(65.62 \%), 25-30$ $(20.83 \%)$, and 30 plus $(9.38 \%)$; this is consistent, to a large extent, with that of Weibo users in general [33]. A majority $(94.79 \%$ or 91 out of 96 ) of the subjects had a university degree or above, meaning that they were likely good informants about the nature of discontinuance in the context of Sina Weibo. Over half of the subjects (50) were non-students with various social economic statuses. Regarding usage experiences, 69 subjects $(71.7 \%)$ had previously used Weibo for more than two years.

\subsection{Data coding procedures}

Two graduate students majored in information systems were recruited and trained as coders. The coding process was a mixture of structured (using a predefined codebook) and open coding. An initial codebook was developed on the basis of factors identified in the literature. The coders were trained to get familiar with the meaning of every factor and the content analysis technique. After several training sessions, both coders reported being confident with the coding analysis. They were then asked to code the textual descriptions of the interviewees independently. Factors defined in the codebook were used wherever applicable; in cases where the coder found difficult to use any predefined code, he/she developed a new code and updated the codebook accordingly. One of the authors acted as a third auditor of the coding process. He/she met the coders for several times at different stages of the coding process to compare their independent codes, discuss inconsistencies, and update the codebook. The coding consistency between the two coders was very high; essentially, they agreed upon over $90 \%$ of the codes.

\section{Result}

The 96 subjects in our sample reported 124 coding units, as one subject could report multiple reasons. The unit of analysis refers to the phrases in each respondent's comments. These coding units were classified into 10 categories and 14 subcategories of factors, which belonged to four general classes including user-related, context-related, function-related, and content-related. Table 2 presents a description of the results, including the categories and sub-categories, the number and relative frequency of responses in each category and sub-category, and exemplary responses. This addresses our RQ1.

To address RQ2, we compared the relative frequency of the reasons for Weibo discontinuance between permanent and intermittent discontinuers using Chi-square tests. The results are summarized in Table 3. Overall, those who left Weibo because of function- or content-related reasons (e.g., system shortcomings, relative disadvantages, and low content quality) were more likely permanent discontinuers; whereas, discontinuers who left for context-related factors were more likely to readopt; the effects of user-related factors are complicated. Below we discuss each (sub-) category of factors in detail.

\subsection{User-related factors}

As Table 2 presented, the most commonly identified factors that precede Weibo discontinuance by the interviewees were those pertaining to themselves, namely, user-related factors (37.90\%); this is consistent with prior research on social network fatigue study [11]. Specifically, $18.55 \%$ of comments stated that users left Weibo because of limited cognitive resources such as time and energy, which has been suggested as an important factor in discontinuance research [14]. The boredom feeling resulted from the lack of social interaction and interest also caused discontinuance $(13.71 \%$ of the comments). In only a few cases $(5.65 \%$ of the comments), participants left Weibo due to low habit.

Table 3 indicates that intermittent discontinuers are more likely to stop using Weibo because of user- 
Table 2. Categories and frequencies of reasons for discontinuance of Weibo

\begin{tabular}{|c|c|c|c|}
\hline Categories & Sub-categories & $\begin{array}{c}\text { Frequency } \\
(\%)\end{array}$ & Examples of Respondents' Comments \\
\hline \multicolumn{2}{|l|}{ User-related } & $47(37.90 \%)$ & \\
\hline \multirow{2}{*}{$\begin{array}{l}\text { Limited } \\
\text { cognitive } \\
\text { resources }\end{array}$} & Busy life & $20(16.13 \%)$ & $\begin{array}{l}\text { My work was very busy so that I had no time to use this } \\
\text { social medium. }\end{array}$ \\
\hline & Usage exhaustion & $3(2.42 \%)$ & Weibo was really time-consuming. \\
\hline \multirow[t]{2}{*}{ Boredom } & Lack of interest & $14(11.29 \%)$ & After a period of use, I felt it not interesting to use Weibo. \\
\hline & $\begin{array}{l}\text { Lack of social } \\
\text { interaction }\end{array}$ & $3(2.42 \%)$ & $\begin{array}{l}\text { I did not interact with my friends in Weibo and there were } \\
\text { no fans that followed me. }\end{array}$ \\
\hline \multicolumn{2}{|l|}{ Low habit } & $7(5.65 \%)$ & I used Weibo at a low frequency and always forgot to use it. \\
\hline \multicolumn{2}{|c|}{ Context-related } & $39(31.45 \%)$ & \\
\hline \multicolumn{2}{|c|}{ Social influences } & $16(12.90 \%)$ & Most of my friends left Weibo, so did I. \\
\hline \multicolumn{2}{|c|}{ Facilitating conditions } & $16(12.90 \%)$ & $\begin{array}{l}\text { When I went home for summer vacation (nearly two } \\
\text { months), there was no wireless to connect with a network. }\end{array}$ \\
\hline \multicolumn{2}{|c|}{ Attractive alternatives } & $7(5.65 \%)$ & $\begin{array}{l}\text { It was more convenient to keep contact with my friends } \\
\text { through WeChat than Weibo. }\end{array}$ \\
\hline \multicolumn{2}{|c|}{ Function-related } & $21(16.94 \%)$ & \\
\hline \multicolumn{2}{|c|}{ Relative disadvantages } & $14(11.29 \%)$ & $\begin{array}{l}\text { Compared with WeChat, Weibo had a terrible privacy } \\
\text { protection and it was not convenient to communicate with } \\
\text { friends at real time. }\end{array}$ \\
\hline \multicolumn{2}{|c|}{ System shortcomings } & $7(5.65 \%)$ & $\begin{array}{l}\text { The system was too complex to use and it occupied large } \\
\text { memory of my smart phone. }\end{array}$ \\
\hline \multicolumn{2}{|c|}{ Content-related } & $17(13.71 \%)$ & \\
\hline \multirow{3}{*}{$\begin{array}{l}\text { Low content } \\
\text { quality }\end{array}$} & Low relevance & $10(8.06 \%)$ & Weibo could not provide the information that I cared about. \\
\hline & Low credibility & $2(1.61 \%)$ & There was so much false information spreading in Weibo. \\
\hline & $\begin{array}{l}\text { Content } \\
\text { redundancy }\end{array}$ & $1(0.81 \%)$ & The information had been always repeated in Weibo. \\
\hline \multicolumn{2}{|c|}{ Over-commercialization } & $4(3.23 \%)$ & I was disgusted with so much advertising existed in Weibo. \\
\hline \multicolumn{2}{|l|}{ Total } & 124 & \\
\hline
\end{tabular}

related factors than permanent discontinuers in general. However, this is not necessarily true for all the constructs within this category. For instance, discontinuers who left for limited cognitive resources stand a bigger chance to readopt the social medium; whereas those who discontinued using Weibo for boredom are almost equally likely to leave forever as to get back. Further, low habit is the only factor for which there existed a significant difference between these two types of discontinuers $(\mathrm{p}<0.05)$; that is, individuals who discontinued using Weibo for low habit are less likely to come back than not.

\subsection{Context-related factors}

Context-related factors may lead to user discontinuance. Context refers to external factors apart from the users, system and task. In this study, context-related factors involved social influences
(12.90\%), facilitating conditions (12.90\%) and attractive alternatives (5.65\%).

As shown in Table 3, compared to permanent discontinuers (19.05\%), more intermittent discontinuers (44.26\%) left Weibo for context-related factors. Although no significant group difference has been found regarding social influence and attractive alternatives, facilitating conditions were related more to intermittent discontinuance than to permanent discontinuance. That is, discontinuers who left Weibo for the lack of facilitating conditions were more $(p<$ $0.05)$ likely to readopt the social medium again after a period of break, once the facilitating conditions are recovered.

\subsection{Function-related factors}

Some participants reported annoyance over the function of the application, which ultimately resulted 
Table 3. Categories and sub-categories of reasons for discontinuance

\begin{tabular}{|c|c|c|c|c|c|c|}
\hline \multirow{2}{*}{$\begin{array}{l}\text { Categories and Sub-categories } \\
\text { of Reasons } \\
\text { User-related } \\
\end{array}$} & \multicolumn{2}{|c|}{$\begin{array}{l}\text { Permanent } \\
\text { Discontinuance (39) }\end{array}$} & \multicolumn{2}{|c|}{$\begin{array}{l}\text { Intermittent } \\
\text { Discontinuance (57) }\end{array}$} & \multirow[t]{2}{*}{ Chi-square } & \multirow[t]{2}{*}{ P value } \\
\hline & 20 & $31.75 \%$ & 27 & $44.26 \%$ & & \\
\hline Limited cognitive resources & 6 & $9.52 \%$ & 17 & $27.87 \%$ & 2.65 & 0.104 \\
\hline Busy life & 5 & $7.94 \%$ & 15 & $24.59 \%$ & 2.557 & 0.110 \\
\hline Usage exhaustion & 1 & $1.59 \%$ & 2 & $3.28 \%$ & 1.398 & 0.237 \\
\hline Boredom & 8 & $12.70 \%$ & 9 & $14.75 \%$ & 0.078 & 0.780 \\
\hline Lack of interest & 6 & $9.52 \%$ & 8 & $14.75 \%$ & 0.034 & 0.854 \\
\hline Lack of social interaction & 2 & $3.17 \%$ & 1 & $1.64 \%$ & 0.871 & 0.351 \\
\hline Low habit & 6 & $9.52 \%$ & 1 & $1.64 \%$ & 6.364 & 0.012* \\
\hline Context-related & 12 & $19.05 \%$ & 27 & $44.26 \%$ & & \\
\hline Social influences & 8 & $12.70 \%$ & 8 & $13.11 \%$ & 0.700 & 0.403 \\
\hline Facilitating conditions & 3 & $4.76 \%$ & 13 & $21.31 \%$ & 3.993 & $0.046 *$ \\
\hline Alternative attraction & 1 & $1.59 \%$ & 6 & $9.84 \%$ & 2.172 & 0.141 \\
\hline Function-related & 19 & $30.16 \%$ & 2 & $3.28 \%$ & & \\
\hline Relative disadvantage & 14 & $22.22 \%$ & 0 & $0.00 \%$ & 23.955 & $0.000 * * *$ \\
\hline System shortcoming & 5 & $7.94 \%$ & 2 & $3.28 \%$ & 2.970 & $0.085 \#$ \\
\hline Content-related & 12 & $19.05 \%$ & 5 & $8.20 \%$ & & \\
\hline Low content quality & 10 & $15.87 \%$ & 3 & $4.92 \%$ & 8.213 & $0.004 * *$ \\
\hline Low relevance & 7 & $11.11 \%$ & 3 & $4.92 \%$ & 3.993 & $0.046^{*}$ \\
\hline Low credibility & 2 & $3.17 \%$ & 0 & $0.00 \%$ & 2.985 & $0.084 \#$ \\
\hline Content redundancy & 1 & $1.59 \%$ & 0 & $0.00 \%$ & 1.477 & 0.224 \\
\hline Over-commercialization & 2 & $3.17 \%$ & 2 & $3.28 \%$ & 0.152 & 0.697 \\
\hline Total Items & 63 & & 61 & & & \\
\hline
\end{tabular}

in discontinuance. Function-related factors refer to relative disadvantages (11.29\%) and system shortcomings $(5.65 \%)$, which users perceived as not catering to their requirements or preferences.

It is notable in Table 3 that most of the discontinuers who stopped using Weibo due to function-related factors belonged to permanent discontinuers. What's more, the number of permanent discontinuers who reported this category of factors was significantly larger than that of intermittent discontinuers. In general, those who were dissatisfied with the functions of the system, particularly in terms of relative disadvantages $(p<$ $0.001)$ and system shortcomings $(p<0.1)$, had a high tendency to discontinue permanently.

\subsection{Content-related factors}

Content is pivotal to Weibo since it may cause frustration, boredom or even anger when it fail to meet users' expectations or interests about content [27], which was shown by our results to be an important factor leading to discontinuance. The comments related to content indicated that some users discontinued using Weibo mainly due to low content quality (10.48\%), which was made up with low relevance, low credibility, and content redundancy. Besides, over-commercialization (3.23\%) also caused discontinuance. From the percentage of each sub-category, discontinuers care more about relevance than credibility and content redundancy.

Table 3 shows that low content quality $(p<0.01)$, especially in terms of relevance $(p<0.05)$ and credibility $(p<0.1)$, was more likely to result in permanent than intermittent discontinuance.

\section{Discussion}

Extending the concept of discontinuance to intermittent discontinuance, this study identifies the reasons for intermittent and permanent discontinuance, and classifies these reasons into four general categories: user-, context-, function- and content-related. Furthermore, we show that although some reasons are common for both intermittent and permanent discontinuance, other factors are unique reasons for either intermittent or permanent discontinuance.

Specifically, the results are complicated for userrelated factors. On the one hand, subjects who stop using Weibo for low habit have a significant 
tendency to leave the social medium forever. This is because a habit of use cannot be developed without repeated use of a specific system [36]. Thus, if users had a low habit of use before discontinuance, increasing his/her usage levels afterwards is hard to imagine. On the other hand, the discontinuance because of limited cognitive resources may be more likely to be intermittent. This is because cognitive resources are volatile and unreliable. After a period of break, subjects may be free and the usage exhaustion will disappear, making discontinuers adopt the social medium again.

As for context-related factors, since the discontinuance due to facilitating conditions is unlikely a result of respondents' voluntariness, it is normal that the discontinuers come back when the situation changes. Besides, the percentage of the two types of discontinuers who leave for social influences are similar, which means that users have the same probabilities to re-adopt the innovation as to leave it forever; this aligns with prior research [37].

It is interesting to find different effects of relative disadvantages vs. alternative attraction, given that both of these factors refer to the presence of competing alternatives. According to the results, relative disadvantage is tightly related to permanent discontinuance while alternative attraction is not. One explanation of this finding is that users will not leave an innovation completely even when a good alternative appears until they find disadvantages and/or shortcomings of the innovation itself. Future research can further validate this theory.

Regarding content-related factors, when subjects are dissatisfied with the content quality (especially in terms of content relevance and credibility), they will make the decision of permanent discontinuance. Other content characteristics (e.g., redundancy) are much less important in triggering discontinuance.

Taken together, maintaining good functionality and task performance of an innovation, and nurturing user habits are critical in preventing permanent discontinuance. As a comparison, discontinuance triggered by contextual factors such as facilitating conditions tends to be intermittent and should not be a major concern to practitioners. These findings have important implications for both research and practice, as elaborated below.

\subsection{Implications for research}

First, this study clarifies the concepts of intermittent and permanent discontinuance, which enriches the literature on social media discontinuance behavior. Specifically, discontinuance behavior cannot be simply regarded as the contrary of continuance behavior due to its uniqueness and complexity. Nor should it be simply viewed as a onetime decision of abandoning an information system at the end of its life cycle. Rather, discontinuance can refer to just one phase in the post-adoption stage.

Second, this study can serve as the basis of future research on the antecedents of discontinuance. We have systematically examined the reasons of discontinuance in the context of Weibo from four categories (user-, context-, function- and contentrelated factors). Researchers in this domain can benefit from our results to build a deep understanding of the reasons underlying discontinuance behaviors.

More importantly, this study explored the commonalities and differences in reasons underlying intermittent vs. permanent discontinuance. By considering the two types of discontinuance simultaneously, we are able to know the importance of function-related factors (e.g., relative disadvantages) and task-related factors (e.g., low content quality) in predicting users' subsequent likelihood of permanent discontinuance. In general, the findings of this research provide a starting point for future research on the two types of discontinuance.

\subsection{Implications for practice}

From a practical perspective, the results imply that social media service providers should not simply view all discontinuers as the same or use the same coping strategy for all discontinuers. This research evidently shows that some discontinuers would come back to the service after taking a break while others may not. The reasons for which they discontinued initially matter.

If the discontinuance was initially due to function- or content-related factors, the discontinuers may be unlikely to come back (i.e., disenchantment discontinuance is often permanent). Luckily, these function- or content-related factors are generally controllable by the social media operators. Thus, it may be wise for them to attend to these factors and take them seriously. Specifically, service providers should be dedicated to the improvement of the system functions to ensure that it operates normally. Moreover, enhancing content supervision is likely beneficial for improving content quality and reducing permanent discontinuance. Further, social media service provides can offer incentives to encourage new users to frequently log in the social medium platform, thereby cultivating user habit which can improve users' dependency on the platform.

As for intermittent discontinuance, social media operators would be happy to know that some discontinuers may re-adopt in the future, especially if 
the initial discontinuance was due to external reasons, which are not very controllable by the service providers. That being said, social media service providers can still take some actions to reduce intermittent discontinuance and encourage readoption after discontinuance. For instance, they can improve their services by reducing the cognitive burdens to use the services.

Our research findings also have important implications towards advertising and social commerce within social media. Most users are disgusted with the phenomenon of overcommercialization, especially when ads are always repeated. Thus, it would be better if advertisers could put on advertisements moderately and precisely in order to avoid users' resentment.

\subsection{Limitations and future studies}

As with any other research, this research has several limitations that may generate directions for future studies. First, we conduct this research in China (a developing country) so that cautions are needed to generalize our findings to Western or developed countries [36]. Users' discontinuance intention and behavior are likely influenced by cultural differences; for example, users in China may be more vulnerable to social influences due to its collectivist culture, whereas Western users may rely more on user-related factors [35]. Future research should explore these factors in other cultures and societies and compare their results with ours, thus adding robustness to our research results and improving the generalizability.

Second, the study treats people who currently do not use Weibo as permanent discontinuers. However, some of them may readopt Weibo in the future. It is difficult (if not impossible) to predict whether a person will reuse a system or not in the future. But in our case, most of the subjects classified as permanent discontinuers had stopped using Weibo for at least six months and expressed low levels of re-adoption intention; it's very unlikely that they would come back after such a long period of time [28]. Future research can address this issue more directly, e.g., by measuring re-adoption intention.

Finally, this study focuses on identifying the reasons of user discontinuance in the context of Weibo, and comparing the reasons for intermittent vs. permanent discontinuance. Given the exploratory nature of this study, we only use qualitative data to obtain various factors that promoted discontinuance behaviors but do not study the inter-relationships among those factors. Yet, it's possible that some factors (e.g., boredom) are closer predictors of discontinuance than, and related to, other factors (e.g., lack of interesting events or connection with friends, low content quality, etc.). Future research can further explore these relationships and draw conclusions through quantitative analysis.

\section{References}

[1] S.Z.M. Din, R.H.M. Anuar, N. Omar, H. Omar, and J.M. Dahlan, "Discovering the Use of Online Recruitment via Social Media of Student Internship" , Procedia Economics \& Finance, 2015 (30), pp. 856-860.

[2] Jankauskaite, and Dovilè, "Social Media as a Tool for Improving Teaching and Learning Experience", Signum Temporis, 2015, 7 (1), pp. 54-59.

[3] J.C. Cortizo, F.M. Carrero, and J.M. Gomez, "Introduction to the Special Issue: Mining Social Media", International Journal of Electronic Commerce, 2011, 15 (3), pp. 5-8.

[4] T. Liang, Y. Ho, Y. Li, and E. Turban, "What Drives Social Commerce: The Role of Social Support and Relationship Quality", International Journal of Electronic Commerce, 2011, 16 (2), pp. 69-90.

[5] T. Liang, and E. Turban, "Introduction to the Special Issue Social Commerce: A Research Framework for Social Commerce", International Journal of Electronic Commerce, 2011, 16 (2), pp. 5-14.

[6] A.T. Stephen, and O. Toubia, "Deriving Value from Social Commerce Networks", Journal of Marketing Research, 2009, 47 (2), pp. 215-228.

[7] Statistical Reports on the Internet Development in China, in: C.I.N.I.C. (CNNIC), (Ed.), Beijin, 2012.

[8] Savannah, "When the Dark Side of Post-adoptive Use Leads to IT Discontinuance: An Exploration of the Role of Intervention", 20th Americas Conference on Information Systems, AMCIS 2014, Association for Information Systems, GA, United states, August 7 - August 9, 2014.

[9] T. Chesney, and S. Lawson, "Critical Mass and Discontinued Use of Social Media", Systems Research and Behavioral Science, 2015, 32 (3), pp. 376-387.

[10] O. Toubia, and A.T. Stephen, "Intrinsic vs. ImageRelated Utility in Social Media: Why Do People Contribute Content to Twitter?", Marketing Science, 2013, 32 (3), pp. 368-392.

[11] J. Zhu, and Z. He, "Internet Diffusion Situation and Prospects in China: A Comparative Study of Beijing, Guangzhou and Hong Kong in 2000", Journalism Bimonthly, 2002 (72), pp. 25-34. (in Chinese)

[12] M. Zhang, and Y. Ye, "Intermittently Discontinued Adoption in the Diffusion of Innovative Communication Technologies: Evidence from National Survey Results in the U.S. and Mainland China", Journalism \& Communication, 2014 (6), pp. 78-98. (in Chinese)

[13] X. Shen, and Y. Li, "An Empirical Investigation of Factors Affecting Smart Health Device Users' Intermittent Discontinuance", Management Science, 2017, 30 (1), pp. 31-42. (in Chinese)

[14] C. York, and J. Turcotte, "Vacationing from Facebook: Adoption, Temporary Discontinuance, and Readoption of 
an Innovation", Communication Research Reports, 2015, 32 (1), pp. 54-62.

[15] E.M. Rogers, Diffusion of Innovations: Modifications of a Model for Telecommunications, Springer Berlin Heidelberg, 1995.

[16] B. Furneaux, and M. Wade, "An Exploration of Organizational Level Information Systems Discontinuance Intentions", MIS Quarterly, 2011, 35 (3), pp. 573-598.

[17] J. Recker, "Towards a Theory of Individual-level Discontinuance of Information Systems Use", International Conference on Information Systems, 2014.

[18] E.M. Rogers, Diffusion of Innovations, Free Press, 2003.

[19] T. Ravindran, A.C.Y. Kuan, and D.G.H. Lian, "Antecedents and Effects of Social Network Fatigue", Journal of the Association for Information Science and Technology, 2014, 65 (11), pp. 2306-2320.

[20] S. Zhang, L. Zhao, Y. Lu, and J. Yang, "Do You Get Tired of Socializing? An Empirical Explanation of Discontinuous Usage Behaviour in Social Network Services", Information \& Management, 2016, 53 (7), pp. 904-914.

[21] C.K. Coursaris, Y. Yun, and J. Sung, "Twitter Users vs. Quitters: A Uses and Gratifications and Diffusion of Innovations Approach in Understanding the Role of Mobility in Microblogging", Ninth International Conference on Mobile Business and 2010 Ninth Global Mobility Roundtable, 2010, pp. 481-486.

[22] O. Turel, "Quitting the Use of a Habituated Hedonic Information System: a Theoretical Model and Empirical Examination of Facebook Users", European Journal of Information Systems, 2015, 24 (4), pp. 431-446.

[23] B. Furneaux, and M. Wade, "The End of the Information System Life: A Model of IS Discontinuance", ACM SIGMIS Database, 2010, 41 (2), pp. 45-69.

[24] B. Furneaux, "Time for a Change: An Exploration of Information Systems Discontinuance", Reaching New Heights. Americas Conference on Information Systems, Keystone, Colorado, USA, August, 2007.

[25] C. Pollard, "Exploring Continued and Discontinued Use of IT: A Case Study of OptionFinder, a Group Support System", Group Decision and Negotiation, 2003, 12 (3), pp. 171-193.

[26] S. Zhang, L. Zhao, Y. Lu, and J. Yang, "Get Tired of Socializing as Social Animal? An Empirical Explanation on Discontinuous Usage Behavior in Social Network Services". Proceedings of PACIS 380, 2015.

[27] C. Maier, S. Laumer, C. Weinert, and T. Weitzel, "The Effects of Technostress and Switching Stress on Discontinued Use of Social Networking Services: A Study of Facebook Use", Information Systems Journal, 2015, 25 (3), pp. 275-308.

[28] I. Cho, "Facebook Discontinuance: Discontinuance as a Temporal Settlement of the Constant Interplay between Disturbance and Coping". Quality \& Quantity, 2015, 49(4), pp. 1531-1548.

[29] C. Maier, S. Laumer, A. Eckhardt, and T. Weitzel, "Giving Too Much Social Support: Social Overload on Social Networking Sites", European Journal of Information System, 2015, 24 (5), pp. 447-464.
[30] M. Parthasarathy, A. Bhattacherjee, "Understanding Post-Adoption Behavior in the Context of Online Services". Information Systems Research, 1998, 9(4): pp. 362-379.

[31] R.A. Gokhale, and R.S Narayanaswamy, "The Role of Experience in Discontinuance of IT Innovations", Proceedings of the 2006 Southern Association for Information Systems Conference, January, 2006.

[32] Z. Guo, Z. Li, and H. Tu, "Sina Microblog: An Information-Driven Online Social Network", 2011, pp. 160-167.

[33] Statistical Reports on Sina Weibo User Development, Sina Weibo Data Center, 2015.

[34] T. Zhou, and H. Li, "Understanding Mobile SNS Continuance Usage in China from the Perspectives of Social Influence and Privacy Concern", Computers in Human Behavior, 2014, 37, pp. 283-289.

[35] A. Bhattacherjee, and C. Lin, "A Unified Model of IT Continuance: Three Complementary Perspectives and Crossover Effects", European Journal of Information Systems, 2015, 24 (4), pp. 364-373.

[36] K.B. Murray, and G. Haubl, "Explaining Cognitive Lock-In: The Role of Skill-Based Habits of Use in Consumer Choice", Journal of Consumer Research, 2007, 34(1), pp. 77-88.

[37] H.C. Kelman, "Compliance, Identification, and Internalization: Three Processes of Attitude Change", Journal of Conflict Resolution, 1958, 2(1), pp. 51-60. 